DESY 98-150; hep-ph/9809277

\title{
Hard Exclusive Electroproduction of Pseudoscalar Mesons and QCD Axial Anomaly
}

\author{
Michael I. Eides * \\ Department of Physics, Pennsylvania State University, University Park, PA 16802, USA, \\ and Petersburg Nuclear Physics Institute, Gatchina, St.Petersburg 188350, Russia \\ Leonid L. Frankfurt ${ }^{\dagger}$ \\ School of Physics and Astronomy, Raymond and Beverly Sackler Faculty of Exact Sciences, \\ Tel Aviv University, Ramat Aviv 69978, Israel, \\ and \\ Mark I. Strikman ${ }^{\ddagger}$ \\ Department of Physics, Pennsylvania State University, University Park, PA 16802, USA, \\ and Deutsches Elektronen Synchrotron DESY, Germany ${ }^{\S}$
}

(September, 1998)

\begin{abstract}
Hard exclusive electroproduction of $\pi^{0}, \eta, \eta^{\prime}$ mesons by the longitudinal photons is considered. With the help of the QCD factorization theorem we show that at sufficiently large virtualities $Q^{2}$ amplitudes of hard exclusive processes may be calculated in terms of the target parton distributions and the minimal Fock component of the pseudoscalar meson light cone wave function at zero interquark distances. This result justifies our calculation of the ratios of the $\pi^{0}: \eta: \eta^{\prime}$ yields for the electroproduction processes off nucleons and deuterons based on the low energy QCD chiral dynamics, and the QCD
\end{abstract}

\footnotetext{
*E-mail address: eides@phys.psu.edu, eides@lnpi.spb.su

${ }^{\dagger}$ E-mail address: frankfur@lev.tau.ac.il

$\ddagger$ E-mail address: strikman@phys.psu.edu

$\S$ On leave of absence from PSU.
} 
anomaly. Thus investigation of these ratios gives a new way to probe the physics of the spontaneous chiral symmetry breaking.

PACS numbers: 13.60 Le 


\section{INTRODUCTION}

Recently the QCD factorization theorem has been generalized to a large group of hard exclusive processes [1], see Eq. (1) below. On the experimental end, a group of hard exclusive high-energy processes was discovered at HERA and FNAL. Their properties agree with the hard QCD expectations but they are distinctively different from those observed in soft high energy processes, see [2] for recent summary and references. Discovery of these processes opened a new frontier to probe the physics of the quark bound states and basic properties of QCD. The aim of this paper is to demonstrate that exploration of the processes of hard exclusive electroproduction of pseudoscalar mesons off a hadron target gives a new way to probe the physics of the spontaneous chiral symmetry breaking.

Wealth of experimental data on the low-energy hadron dynamics was understood already before advent of QCD in terms of the current algebra and chiral Lagrangians (see, e.g., review in [3]). After emergence of QCD as the theory of strong interactions it turned out that predictions of the current algebra and the chiral Lagrangians could be more or less rigorously derived from the microscopic theory of strong interactions, if one assumes that the inherent chiral symmetry of QCD with massless quarks is spontaneously broken in the ground state. This bright picture was marred by the fact that naively spontaneous breaking of the chiral symmetry in QCD predicts a pattern of masses of the neutral pseudoscalar mesons $\pi, \eta, \eta^{\prime}$ which contradicts the experimental data [4]. This problem of the mass spectrum of the neutral pseudogoldstone bosons (and also of the large partial width of the decay $\eta \rightarrow 3 \pi$ )is referred to as the $U_{1}$ problem. Resolution of the $U_{1}$ problem was triggered by the remark by t'Hooft [5] that due to the Adler-Bell-Jackiw anomaly [6] the singlet axial current in QCD is non conserved and thus there could be a nontrivial contribution to the mass of the respective would be Goldstone boson. Such additional contribution to the boson mass could be generated for example by the instanton contribution to the axial anomaly. A practical way to obtain a heavy $\eta^{\prime}$ in QCD with large number of colors $N_{c}$ was suggested by Witten [7]. It is important to realize that the very existence of the axial anomaly is insufficient for resolution of the $U_{1}$ problem. Veneziano [8] has demonstrated that one has to assume existence of an additional massless pole (so called ghost pole) in the correlators of the certain gauge-noninvariant gluon currents. It was checked a long time ago that the ghost pole hypothesis is self-consistent and provides a satisfactory explanation of the spectrum of the low-lying pseudoscalar mesons, of the large width of the decay $\eta \rightarrow 3 \pi$, the widths of the decays $\eta, \eta^{\prime} \rightarrow 2 \gamma$, and successfully describes also a number of other low-energy reactions [9].

We will show in this paper that the ghost pole hypothesis leads to observable phenomena not only in the realm of the low-energy physics, but also in the hard exclusive electroproduction of pseudoscalar mesons. This means that one can probe the physics of the spontaneous chiral symmetry breaking in hard exclusive processes. We will obtain a number of predictions for the ratios of certain cross sections for hard exclusive processes in terms of the parameters derived from the low-energy ghost-pole physics.

The key observation which leads to connection between the hard diffractive electroproduction and the parameters of the low-energy physics was made in [1], where the QCD factorization theorem was proven for the forward hard exclusive electroproduction of mesons by longitudinally polarized photons 


$$
\gamma_{L}^{*}(q)+p \rightarrow M(q+\Delta)+B^{\prime}(p-\Delta)
$$

at large $Q^{2}$, with $t$ and $x=Q^{2} / 2 p \cdot q$ fixed. It asserts that the electroproduction amplitude has the form

$$
\begin{aligned}
& \sum_{i} \int_{0}^{1} d z \int d \xi f_{i / p}(\xi, \xi-x ; t, \mu) H_{i M}\left(\xi / x, Q^{2}, z, \mu\right) \phi_{M}\left(z, Q^{2}, \mu^{2}\right) \\
& + \text { power-suppressed corrections, }
\end{aligned}
$$

where f's are skewed (off(non)-diagonal(forward)) parton helicity densities within a nucleon, $\phi$ is the light-cone meson distribution amplitude [10], $z$ is the light-cone fraction of the photon momentum carried by a quark (antiquark), and $H$ is a hard-scattering coefficient, which may be calculated in the form of the expansion in powers of the strong coupling constant $\alpha_{s}(Q)$. The scale $\mu^{2}$ in Eq. (2) as usual for the factorization relations is $\sim Q^{2}$ hence there is a direct relation between meson wave functions probed in hard exclusive two-body processes and in form factor physics.

Note that for sufficiently small $x \leq 0.1$ and large $Q^{2}$ the skewed distributions can be expressed through the diagonal distributions at large $x$ and $Q^{2} \sim$ few $\mathrm{GeV}^{2}$ [11, 12]. Relation between skewed and usual parton densities at moderately small $x$ has been discussed in 13. At the same time for $x \geq 0.1$ and $Q^{2} \sim$ few $\mathrm{GeV}^{2}$ calculations of the skewed distributions within the chiral model were recently performed in [14].

Moreover these skewed parton densities really will not enter into our final expressions for the ratios of cross sections since they are almost completely canceled out in these ratios. This cancellation is especially precise in the case of the target with vanishing isospin, namely for the deuteron target. We neglect here the contribution of the difference between the $s$ and $\bar{s}$ distributions within a nucleon target. Thus for large $Q^{2}$ only the integral

$$
\int \frac{d z}{z(1-z)} \phi_{M}\left(z, Q^{2}, \mu^{2}=Q^{2}\right)
$$

of the light-cone meson distribution amplitude $\phi_{M}\left(z, Q^{2}, \mu^{2}\right)$ enters in the expressions for the ratios of cross sections of electroproduction of pseudoscalar mesons.

At the same time it is well known that the meson distribution amplitude is completely determined by the conformal invariance of QCD and the quantum numbers of the meson $M$ and can be decomposed in a series over the Gegenbauer polynomials $C_{n}^{\frac{3}{2}}$ [10,15, 16, In the limit $Q^{2} \rightarrow \infty$ only the leading term with $n=0$ survives and

$$
\phi_{M}\left(z, Q^{2}, \mu^{2}=Q^{2}\right)=c_{M} z(1-z)
$$

As a result in the case of pseudoscalar mesons integration over $z$ in the expression for the amplitude becomes trivial and the amplitude in Eq.(3) is simply proportional to $c_{M}$. Hence, all nontrivial dynamical information about the amplitude is contained in this case in the normalization factor of the light-cone wave function which is equal to the matrix element of the local axial current 10,15, 16]. In this respect calculation of ratios of hard diffractive pseudoscalar meson production cross sections is theoretically even more transparent than calculation of absolute cross sections at moderate $Q^{2}$, when respective integrals of the meson 
wave function are more complicated and do not reduce to matrix elements of a local operator |11||.

Let us emphasize once again that the same matrix element of the axial current determines both the amplitudes of the low-energy interaction of the pseudoscalar mesons and the amplitudes of the hard diffractive electroproduction of these mesons. Thus the hard diffractive processes can be used to investigate intricate properties of the physics of spontaneously broken chiral symmetry in QCD.

\section{SPIN DEPENDENCE OF THE AMPLITUDES FOR PSEUDOSCALAR MESON ELECTROPRODUCTION IN QCD}

Quark-antiquark pairs in hard exclusive processes initiated by longitudinally polarized photons are produced at small relative transverse distances $\propto 1 / Q$. Thus such processes probe interaction of a small size $q \bar{q}$ pair with a hadron target. Localization of the color charge in a small volume justifies applicability of the perturbative QCD for the description of such processes. Thus to describe hard exclusive electroproduction of pseudoscalar mesons it is necessary to calculate the handbag diagram. Convenient technology of calculation is to evaluate the imaginary parts of the amplitudes and then to reconstruct the real part using the dispersion representation of the amplitude over energy. Simplification arises since the amplitude corresponding to the negative charge parity in the crossed channel has no subtraction constants and is unambiguously determined by the dispersion relations. The gluons which are produced in the intermediate state are predominantly transversely polarized relative to the reaction axis defined by the photon and the target momenta. It is convenient to use the Fierz transformation to factorize the spin structure of the amplitudes $\sum_{\mu=1,2} \gamma_{\mu} \otimes$ $\gamma_{\mu}=2 \gamma_{5} \otimes \gamma_{5}+\gamma_{\mu_{\|}} \gamma_{5} \otimes \gamma_{\mu_{\|}} \gamma_{5}+\ldots$. We omit terms which do not give contribution to the

${ }^{1}$ It is worth noting that experimental investigation of the ratios of the yields of different mesons has another important advantage. Indeed, it was demonstrated in [17] that up to rather large $Q^{2}$ the absolute cross sections are suppressed significantly due to the higher twist effects originating from comparable transverse size of the longitudinal photon and meson wave functions. However the overall transverse size was found to be sufficiently small $\leq 0.4 \mathrm{fm}$ at $Q^{2} \geq 5 \mathrm{GeV}^{2}$. Due to color transparency this leads to a strong suppression of the final state interaction of the $q \bar{q}$ pair which will form the meson and the residual baryon system. For $W \leq 20 G e V$ this cross section is of the order of few $m b$. Besides, the expansion of the $q \bar{q}$ system to a normal hadron size in the nucleon rest frame takes a distance $l_{c o h} \sim p_{M} / \Delta m_{M}^{2}$ where $\Delta m_{M}^{2}$ is characteristic light-cone energy denominator for a meson $M$ which is $\leq 1 \mathrm{GeV}^{2}$, cf. review [18]. One can easily see that condition $l_{\text {coh }} \gg r_{N}$ is satisfied for $x \geq 0.2$ already for $Q^{2} \geq 5 \mathrm{GeV}^{2}$. Hence, it seems likely that a precocious factorization into three blocks - overlap integral, hard blob, skewed density - could be valid already at relatively low $\mathrm{Q}^{2}$ leading to a precocious scaling of the ratios of the cross sections as a function $Q^{2}$. At the same time predictions for the absolute cross sections are valid only for $Q^{2} \geq 10 \mathrm{GeV}^{2}$. In the case of the ratios of the yields of pseudoscalar and vector mesons, like the $\pi^{0} / \rho^{0}$ ratio calculated in [19,20] at large $Q^{2}$ this argument may not work as well due to possible different transverse sizes of $q \bar{q}$ configurations for pseudoscalar and vector mesons. 
box diagram. In the case of forward scattering the first term gives vanishing contribution as a result of chirality conservation. For nonforward scattering when $t$ is not large the second term dominates also since the contribution of the first term to the amplitude of electroproduction of a pseudoscalar meson is suppressed by an additional factor $x$. Thus we have proved that only the spin flip amplitude gives nonvanishing contribution (see also [1.19]). Factorization of the spin structure of the handbag diagram from that in the parton distributions within a target means that the handbag diagram is effectively averaged over the quark helicities. Due to the dominance of the $\gamma_{\mu_{\|}} \gamma_{5}$ term only the component of the meson wave function which is given by the matrix element of the axial current survives in the trace of the handbag diagram after this averaging.

\section{NAIVE $S U(3)$ SYMMETRIC RELATIVE PRODUCTION RATES}

As we have emphasized above the interquark distances in the pseudoscalar mesons probed in hard processes are small. Hence, the decomposition of the hadron state vector in terms of the quark and gluon Fock space states can be mathematically defined and justified within the framework of the QCD factorization theorem. In the case of hard diffractive electroproduction of a meson by longitudinally polarized photons only the quark-antiquark component of the general wave function contributes [21]. Other partons of the light cone wave function of a hadron are included into the structure functions of a target. The amplitude for the exclusive neutral pseudoscalar production off the hadron target in terms of the light cone wave functions were obtained in [1]. Weights of quarks of different flavors in the box diagram are determined by the electromagnetic current operator

$$
j_{\mu}=\frac{2}{3} \bar{u} \gamma_{\mu} u-\frac{1}{3} \bar{d} \gamma_{\mu} d-\frac{1}{3} \bar{s} \gamma_{\mu} s
$$

On the other hand the strength of the pseudoscalar-quark-antiquark vertex is determined, as we have explained above, by the matrix elements

$$
<0\left|\bar{\psi}_{f} \gamma^{\mu} \gamma_{5} \psi_{f}\right| M>=i f_{f} p^{\mu}
$$

where $\psi_{f}$ is a quark field of flavor $f$.

Hence, the helicity distribution functions of the quarks of flavor $f$ enter the production amplitudes with the weights determined by the effective axial current

$$
j_{\mu}^{5}=i\left(\frac{2}{3} \bar{u} \gamma_{\mu} \gamma_{5} u-\frac{1}{3} \bar{d} \gamma_{\mu} \gamma_{5} d-\frac{1}{3} \bar{s} \gamma_{\mu} \gamma_{5} s\right)
$$

or in other words the weights are proportional to the matrix elements in Eq.(6) multiplied by the respective quark electric charges.

Let us consider first the hard exclusive neutral pseudoscalar production in the limit of exact $S U(3)$ symmetry neglecting the axial anomaly. In this case matrix elements of the $S U(3)$ axial currents

$$
<0\left|\frac{\bar{u} \gamma^{\mu} \gamma_{5} u-\bar{d} \gamma^{\mu} \gamma_{5} d}{\sqrt{2}}\right| \pi^{0}>=i f_{\pi} p^{\mu}
$$




$$
\begin{aligned}
& <0\left|\frac{\bar{d} \gamma^{\mu} \gamma_{5} d+\bar{u} \gamma^{\mu} \gamma_{5} u-2 \bar{s} \gamma^{\mu} \gamma_{5} s}{\sqrt{6}}\right| \eta>=i f_{\eta} p^{\mu}, \\
& <0\left|\frac{\bar{d} \gamma^{\mu} \gamma_{5} d+\bar{u} \gamma^{\mu} \gamma_{5} u+\bar{s} \gamma^{\mu} \gamma_{5} s}{\sqrt{3}}\right| \eta^{\prime}>=i f_{\eta^{\prime}} p^{\mu},
\end{aligned}
$$

contain one and the same constant $f_{\pi}=f_{\eta}=f_{\eta^{\prime}} \approx 132 \mathrm{MeV}$ on the right hand side. The matrix elements in Eq.(16) can be easily obtained from the $S U(3)$ relationships

$$
\begin{gathered}
<0\left|\frac{\bar{u} \gamma^{\mu} \gamma_{5} u}{\sqrt{2}}\right| \pi^{0}>=-<0\left|\frac{\bar{d} \gamma^{\mu} \gamma_{5} d}{\sqrt{2}}\right| \pi^{0}>=i \frac{f_{\pi}}{2} p^{\mu}, \\
<0\left|\frac{\bar{d} \gamma^{\mu} \gamma_{5} d}{\sqrt{6}}\right| \eta>=<0\left|\frac{\bar{u} \gamma^{\mu} \gamma_{5} u}{\sqrt{6}}\right| \eta>=-<0\left|\frac{\bar{s} \gamma^{\mu} \gamma_{5} s}{2 \sqrt{6}}\right| \eta>=i \frac{f_{\eta}}{6} p^{\mu}, \\
<0\left|\frac{\bar{d} \gamma^{\mu} \gamma_{5} d}{\sqrt{3}}\right| \eta^{\prime}>=<0\left|\frac{\bar{u} \gamma^{\mu} \gamma_{5} u}{\sqrt{3}}\right| \eta^{\prime}>=<0\left|\frac{\bar{s} \gamma^{\mu} \gamma_{5} s}{\sqrt{3}}\right| \eta^{\prime}>=i \frac{f_{\eta^{\prime}}}{3} p^{\mu} .
\end{gathered}
$$

In this approximation the amplitudes of hard exclusive diffractive electroproduction have the form (we omit the common factor $f_{\pi}$ since we are interested only in the ratios of the amplitudes)

$$
\begin{aligned}
&<0\left|j_{\mu}^{5}\right| \pi^{0}>\sim \frac{1}{\sqrt{2}}\left(\frac{2}{3} \Delta u+\frac{1}{3} \Delta d\right), \\
&<0\left|j_{\mu}^{5}\right| \eta>\sim \frac{1}{\sqrt{6}}\left(\frac{2}{3} \Delta u-\frac{1}{3} \Delta d+\frac{2}{3} \Delta s\right), \\
&<0\left|j_{\mu}^{5}\right| \eta^{\prime}>\sim \frac{1}{\sqrt{3}}\left(\frac{2}{3} \Delta u-\frac{1}{3} \Delta d-\frac{1}{3} \Delta s\right),
\end{aligned}
$$

where $\Delta u, \Delta d$ and $\Delta s$ are the helicity distribution functions of the quarks in the target. Hence in the $\mathrm{SU}(3)$ limit we obtain:

$$
\pi^{0}: \eta: \eta^{\prime}=\frac{1}{2}\left(\frac{2}{3} \Delta u+\frac{1}{3} \Delta d\right)^{2}: \frac{1}{6}\left(\frac{2}{3} \Delta u-\frac{1}{3} \Delta d+\frac{2}{3} \Delta s\right)^{2}: \frac{1}{3}\left(\frac{2}{3} \Delta u-\frac{1}{3} \Delta d-\frac{1}{3} \Delta s\right)^{2},
$$

Assuming that for the proton $\Delta s=0$ and $\Delta d \approx-\Delta u$, we immediately obtain the ratios of production cross sections

$$
\pi^{0}: \eta: \eta^{\prime}=1: 3: 6
$$

which were first obtained in [1]. Above ratios differ from those obtained in [19] within the same approximations. A small uncertainty in the predictions due to unknown value of $\Delta s$ can be further suppressed by selecting $x \geq 0.2$ where the strange sea is surely negligible. 


\section{RELATIVE PRODUCTION RATES WITH ACCOUNT OF THE QCD AXIAL ANOMALY AND $S U(3)$ BREAKING}

Due to the presence of the QCD axial anomaly in the neutral channel, and due to an explicit flavor $S U(3)$ breaking by the current quark masses, the matrix elements of the axial currents in QCD have a more involved structure than in the $S U(3)$ symmetric case considered above. These two effects in the matrix elements were taken into account in [9], and we will use these results in the problem of hard exclusive diffractive production of the pseudoscalar mesons.

The relationships in Eq.(9) remain valid since we still assume $S U(2)$ symmetry and there is no anomaly in the isovector channels. In order to obtain the axial current matrix elements for $\eta$ and $\eta^{\prime}$ we introduce auxiliary currents

$$
\begin{gathered}
j_{\mu 5}^{1}=\frac{\bar{u} \gamma_{\mu} \gamma_{5} u+\bar{d} \gamma_{\mu} \gamma_{5} d}{\sqrt{2}}, \\
j_{\mu 5}^{2}=\bar{s} \gamma_{\mu} \gamma_{5} s .
\end{gathered}
$$

Conservation of these currents is broken by the QCD axial anomaly

$$
\begin{gathered}
\partial_{\mu} j_{\mu 5}^{1}=2 i \frac{m_{u} \bar{u} \gamma_{5} u+m_{d} \bar{d} \gamma_{5} d}{\sqrt{2}}+2 \sqrt{2} Q \equiv P_{1}+2 \sqrt{2} Q \\
\partial_{\mu} j_{\mu 5}^{2}=2 i m_{s} \bar{s} \gamma_{5} s+2 Q \equiv P_{2}+2 Q
\end{gathered}
$$

where $Q=\frac{\alpha_{s}}{8 \pi} G \tilde{G}$.

We need to obtain analogies of the relationships in Eq.(10) and Eq.(11) in the real QCD. As a technical tool we define new constants $f_{\eta\left(\eta^{\prime}\right)}^{1}$ and $f_{\eta\left(\eta^{\prime}\right)}^{2}$ (note that these constants $f_{\eta\left(\eta^{\prime}\right)}^{i}$ do not coincide with any constants in [9])

$$
\begin{aligned}
& <0\left|j_{\mu 5}^{1}\right| \eta>=i f_{\eta}^{1} p^{\mu}, \\
& <0\left|j_{\mu 5}^{1}\right| \eta^{\prime}>=i f_{\eta^{\prime}}^{1} p^{\mu} \\
& <0\left|j_{\mu 5}^{2}\right| \eta>=i f_{\eta}^{2} p^{\mu} \\
& <0\left|j_{\mu 5}^{2}\right| \eta^{\prime}>=i f_{\eta^{\prime}}^{2} p^{\mu}
\end{aligned}
$$

In the end of the day these constants will determine the production amplitudes.

Due to the axial anomaly in Eq.(17) we have

$$
\begin{gathered}
f_{\eta}^{1} m_{\eta}^{2}=<0\left|P_{1}+2 \sqrt{2} Q\right| \eta>, \\
f_{\eta^{\prime}}^{1} m_{\eta^{\prime}}^{2}=<0\left|P_{1}+2 \sqrt{2} Q\right| \eta^{\prime}>,
\end{gathered}
$$




$$
\begin{gathered}
f_{\eta}^{2} m_{\eta}^{2}=<0\left|P_{2}+2 Q\right| \eta>, \\
f_{\eta^{\prime}}^{2} m_{\eta^{\prime}}^{2}=<0\left|P_{2}+2 Q\right| \eta^{\prime}>.
\end{gathered}
$$

Expressions on the right hand side of these equations were calculated in [9] (note that $f_{1}$ and $f_{2}$ in the relationships below are as they are defined in [9] and have nothing to do with the numerous $f$ 's defined above)

$$
\begin{aligned}
& <0|Q| \eta>=\sqrt{\frac{\lambda^{4}\left(m_{2}^{2}-m_{\eta}^{2}\right)\left(m_{\eta}^{2}-m_{1}^{2}\right)}{m_{\eta^{\prime}}^{2}-m_{\eta}^{2}}}, \\
& <0|Q| \eta^{\prime}>=\sqrt{\frac{\lambda^{4}\left(m_{\eta^{\prime}}^{2}-m_{2}^{2}\right)\left(m_{\eta^{\prime}}^{2}-m_{1}^{2}\right)}{m_{\eta^{\prime}}^{2}-m_{\eta}^{2}}}, \\
& <0\left|P_{1}\right| \eta>=\sqrt{\frac{f_{1}^{2} m_{1}^{4}\left(m_{2}^{2}+\mu_{2}^{2}-m_{\eta}^{2}\right)}{m_{\eta^{\prime}}^{2}-m_{\eta}^{2}}}, \\
& <0\left|P_{1}\right| \eta^{\prime}>=\sqrt{\frac{f_{1}^{2} m_{1}^{4}\left(m_{\eta^{\prime}}^{2}-m_{2}^{2}-\mu_{2}^{2}\right)}{m_{\eta^{\prime}}^{2}-m_{\eta}^{2}}} \\
& <0\left|P_{2}\right| \eta>=-\sqrt{\frac{f_{2}^{2} m_{2}^{4}\left(m_{1}^{2}+\mu_{1}^{2}-m_{\eta}^{2}\right)}{m_{\eta^{\prime}}^{2}-m_{\eta}^{2}}} \\
& <0\left|P_{2}\right| \eta^{\prime}>=\sqrt{\frac{f_{2}^{2} m_{2}^{4}\left(m_{\eta^{\prime}}^{2}-m_{1}^{2}-\mu_{1}^{2}\right)}{m_{\eta^{\prime}}^{2}-m_{\eta}^{2}}} .
\end{aligned}
$$
[9]

Now we are in a position to calculate unknown constants $f_{\eta\left(\eta^{\prime}\right)}^{(i)}$. We use parameters from

$$
\begin{gathered}
f_{1}=f_{\pi}=0.132 \mathrm{GeV}, \quad f_{2}=f_{\pi}+2\left(f_{K}-f_{\pi}\right)=0.178 \mathrm{GeV}, \quad \frac{f_{2}}{f_{\pi}}=1.35 \\
\mu_{1}^{2}=0.57 \mathrm{GeV}^{2}, \quad \mu_{2}^{2}=0.16 G e V^{2}, \quad \lambda^{4}=\frac{f_{\pi}^{2} \mu_{1}^{2}}{8}=(0.188 G e V)^{4} \\
m_{\eta}^{2}=0.307 G e V^{2}(0.301), \quad m_{\eta^{\prime}}^{2}=0.912 G e v^{2}(0.917), \\
m_{1}^{2} \approx m_{\pi}^{2} \approx 0.02 G e V^{2}, \quad m_{2}^{2} \approx m_{K^{+}}^{2}+-m_{\pi}^{2} \approx 0.47 G e V^{2},
\end{gathered}
$$


where in the brackets we have written experimental values of the masses.

Note that if one ignores the anomalous contribution and the $S U(3)$ breaking for the constants $f_{i}$ then our new formula immediately reproduce the naive $S U(3)$ symmetric results considered in [1] and reproduced above.

Substituting the numbers above in Eq.(22)-Eq.(25) we obtain the numerical values for the constants $f_{\eta\left(\eta^{\prime}\right)}^{i}$

$$
\begin{aligned}
& f_{\eta}^{(1)}=0.097, \quad f_{\eta^{\prime}}^{(1)}=0.090, \\
& f_{\eta}^{(2)}=-0.122, \quad f_{\eta^{\prime}}^{(2)}=0.129 .
\end{aligned}
$$

Using Eq.(18) we obtain

$$
\begin{aligned}
& <0\left|j_{\mu 5}^{1}\right| \eta>=0.097 i p^{\mu} \\
& <0\left|j_{\mu 5}^{1}\right| \eta^{\prime}>=0.090 i p^{\mu} \\
& <0\left|j_{\mu 5}^{2}\right| \eta>=-0.122 i p^{\mu} \\
& <0\left|j_{\mu 5}^{2}\right| \eta^{\prime}>=0.129 i p^{\mu}
\end{aligned}
$$

or

$$
\begin{gathered}
<0\left|\frac{\bar{u} \gamma_{\mu} \gamma_{5} u}{\sqrt{2}}\right| \eta>=<0\left|\frac{\bar{d} \gamma_{\mu} \gamma_{5} d}{\sqrt{2}}\right| \eta>=\frac{0.097}{2} i p^{\mu}=0.366 i f_{\pi} p^{\mu}, \quad \text { (0.289), } \\
<0\left|\frac{\bar{u} \gamma_{\mu} \gamma_{5} u}{\sqrt{2}}\right| \eta^{\prime}>=<0\left|\frac{\bar{d} \gamma_{\mu} \gamma_{5} d}{\sqrt{2}}\right| \eta^{\prime}>=\frac{0.090}{2} i p^{\mu}=0.343 i f_{\pi} p^{\mu}, \quad \text { (0.408), } \\
<0\left|\bar{s} \gamma_{\mu} \gamma_{5} s\right| \eta>=-0.122 i p^{\mu}=-0.927 i f_{\pi} p^{\mu}, \quad(-0.816), \\
<0\left|\bar{s} \gamma_{\mu} \gamma_{5} s\right| \eta^{\prime}>=0.129 i p^{\mu}=0.980 i f_{\pi} p^{\mu}
\end{gathered}
$$

where the naive values of the coefficients from Eq.(9) are written in the parentheses.

Now we can easily repeat calculation of the production amplitudes and cross sections made above with account of the anomaly and the $S U(3)$ breaking. We obtain instead of Eq.(12) (note that nothing changes for $\pi^{0}$-meson)

$$
\begin{gathered}
<0\left|j_{\mu}^{5}\right| \pi^{0}>\sim \frac{1}{\sqrt{2}}\left(\frac{2}{3} \Delta u+\frac{1}{3} \Delta d\right), \\
<0\left|j_{\mu}^{5}\right| \eta>\sim \frac{2 \sqrt{2}}{3} 0.366 \Delta u-\frac{\sqrt{2}}{3} 0.366 \Delta d+\frac{1}{3} 0.927 \Delta s
\end{gathered}
$$




$$
\begin{gathered}
=\frac{1}{\sqrt{6}}\left(\frac{2}{3} \cdot 1.27 \Delta u-\frac{1}{3} \cdot 1.27 \Delta d+\frac{2}{3} \cdot 1.14 \Delta s\right), \\
<0\left|j_{\mu}^{5}\right| \eta^{\prime}>\sim \frac{2 \sqrt{2}}{3} 0.343 \Delta u-\frac{\sqrt{2}}{3} 0.343 \Delta d-\frac{1}{3} 0.980 \Delta s \\
=\frac{1}{\sqrt{3}}\left(\frac{2}{3} \cdot 0.840 \Delta u-\frac{1}{3} \cdot 0.840 \Delta d-\frac{1}{3} \cdot 1.697 \Delta s\right) .
\end{gathered}
$$

Then for the cross section ratios we have (compare with the $S U(3)$ symmetric result in Eq.(13))

$$
\begin{gathered}
\pi^{0}: \eta: \eta^{\prime}=\frac{1}{2}\left(\frac{2}{3} \Delta u+\frac{1}{3} \Delta d\right)^{2}: \frac{1}{6}\left(\frac{2}{3} \cdot 1.27 \Delta u-\frac{1}{3} \cdot 1.27 \Delta d+\frac{2}{3} \cdot 1.14 \Delta s\right)^{2} \\
: \frac{1}{3}\left(\frac{2}{3} \cdot 0.840 \Delta u-\frac{1}{3} \cdot 0.840 \Delta d-\frac{1}{3} \cdot 1.697 \Delta s\right)^{2} .
\end{gathered}
$$

To analyze the consequences of Eq.(38) we will use the standard assumption that the quark helicity distributions satisfy the relation $\Delta s=0$, see e.g. [23].

First we observe that the $\eta$ production rate is enhanced by a factor of 1.61 and the $\eta^{\prime}$ production is suppressed by a factor of 0.71 in comparison with the naive $S U(3)$ limit, leading to

$$
\eta: \eta^{\prime}=1: 0.87
$$

to be compared with the naive result 1:2 in Eq.(14). Qualitatively this is quite natural since it means enhancement of the $\eta$ coupling with the $u$ and $d$ quarks, and suppression of the $\eta^{\prime}$ coupling with the same quarks. This may be interpreted in the sense that due to mixing with the QCD ghost pole $\eta^{\prime}$-meson contents is enriched by additional glue.

In the case of the coherent scattering off the deuteron large cancellations occur since the amplitudes for scattering off proton and neutron have opposite sign due to $\Delta u \approx-\Delta d$. This indicates that coherent diffraction off the deuteron will be strongly suppressed as compared to the break up channel. There is an additional suppression due to a spin flip in the deuteron vertex. Naive $S U(3)$ symmetric result for the cross sections ratios for the coherent scattering off the deuteron is given by the relationship $\pi: \eta: \eta^{\prime}=27: 1: 2$, while the calculation with account of the chiral anomaly and $S U(3)$ breaking leads to the result

$$
\pi: \eta: \eta^{\prime}=27: 1.6: 1.4
$$

which is markedly different from the case of scattering off the proton as well as off the neutron (which can be studied in the deuteron break up reaction).

For numerical studies of the implications of Eq.(38) for production of $\eta$ and $\pi^{0}$ off nucleons we use the results of the recent global analysis of inclusive and semi-inclusive polarization data [23]. For the first estimate we neglect effects of skewedness of relevant parton densities. For certainty we fix $Q^{2}=20 \mathrm{GeV}^{2}$ and take the fit 1 of the set of fits presented in [23]. We 
checked that our results depend very weakly on $Q^{2}$ scale of the parton densities. This is due to a rather small scaling violation in the valence quark channels. Also we found that different fits of [23] lead to rather small $(\leq 10 \%)$ changes of the considered ratios.

In Fig. 1 we present the ratio of the cross section of production of $\eta$ and $\pi^{0}$ off the proton and neutron targets. In Fig. 2 we compare yields of $\pi^{0}$ and $\eta$ off the proton and neutron targets. One can see that the current data on $\Delta u_{V}, \Delta d_{V}$ lead to a rich pattern of $x$, and target dependencies which would be worth studying experimentally. We also observe that a simplifying approximation $\Delta u_{V} \approx-\Delta d_{V}$ is inadequate for the quantitative predictions.

A set of axial current matrix elements $<0\left|\bar{q} \gamma_{\mu} \gamma_{5} q\right| \eta>,<0\left|\bar{q} \gamma_{\mu} \gamma_{5} q\right| \eta>$ was obtained in a recent phenomenological analysis [24] of the various low energy data on production and decays of $\eta, \eta^{\prime}$ mesons. The numbers derived in [24] differ strongly from the SU(3) expectations, and are pretty close to the ones obtained in [9] from the analysis of the anomalous Ward identities in QCD and used in the present work. The largest difference $(\sim 13 \%)$ between the two sets of matrix elements occurs for the $<0\left|\bar{u} \gamma_{\mu} \gamma_{5} u\right| \eta>$ matrix element. In case of the cross section ratios considered above the phenomenological parameters from [24] would decrease the value of the $\eta^{\prime} / \pi^{0}$ ratio by a factor of .98 and increase the value of the $\eta / \pi^{0}$ ratio by a factor $\sim 1.27$.

Similar matrix elements are relevant also for the description of the processes $\gamma \gamma^{*} \rightarrow$ $\pi^{0}, \eta, \eta^{\prime}$ at large $Q^{2}$ [25]. In the case of the $\pi^{0}$ production the QCD analysis of [26] has provided a good description of the data giving a new evidence for closeness of the $q \bar{q}$ component of the pion wave function to the asymptotic shape. Analysis of the $\eta, \eta^{\prime}$ production performed recently in 24] shows that the large $Q^{2}$ data agree with the results of calculations based on the values of the phenomenological matrix elements obtained in [24]. The matrix elements from [9] lead to the same magnitude of the $\eta^{\prime}$ transition form factor at large $Q^{2}$ and to about $\sim 20 \%$ lower magnitude of the $\eta$ transition form factor. The accuracy of the data is not sufficient yet to discriminate between the two sets of the matrix elements.

\section{DISCUSSION OF RESULTS}

We have demonstrated above that the hard exclusive electroproduction of the pseudoscalar mesons $M$ off a nucleon $\gamma+N \rightarrow M+N$ can be described in the leading twist with the help of the perturbative quark handbag diagram. However, this production process may be also mediated by the odderon exchange [22]. Distinctive feature of the odderon exchange is that the cross section only slowly depends on the photon energy but rapidly decreases as $\alpha_{s}^{3}\left(Q^{2}\right) / Q^{10}$ with increase of the photon virtuality $Q^{2}$. On the other hand contribution to the cross section which is due to the exchange of $q \bar{q}$ pair discussed above behaves itself as

$\alpha_{s}^{2} x^{n} /\left(Q^{6}\right)$, i.e. this contribution decreases with energy. Here, $n$ is equal 2 if the perturbative $q \bar{q}$ pair exchange describes amplitude at large intervals in rapidity. If the $x$ dependence of amplitude due to the q $\bar{q}$ pair exchange is estimated assuming that the $x$ dependence of the nonperturbative support function in the QCD evolution equation is given by the exchange by the vector meson Regge trajectory, then $n \approx 1$.

The ratio of the respective cross sections has the form $c \alpha_{s}\left(Q^{2}\right) / Q^{4} x^{n}$, where $c$ is a model dependent numerical factor. Odderon exchange is a sum of the hard and soft QCD contributions. Even signs of these contributions cannot be derived from the general principles. Moreover, hard contribution to the odderon exchange cannot be expressed through the par- 
ton distributions of a proton since the soft (nonperturbative) end-point contribution in the integration over the fraction of the photon momentum carried by the quark lines is not suppressed in the case of the odderon exchange.

One option to suppress uncontrollable and therefore dangerous odderon contribution is to investigate the ratios at moderate $x$ where the odderon exchange is suppressed. Another option is to consider the processes where the odderon contribution is forbidden by the quantum numbers. A reaction with $\Delta^{+}$production on the proton $\gamma_{L}+p \rightarrow M+\Delta^{+}$is a good example. In this case the odderon exchange is suppressed by the isospin conservation. Another advantage of this reaction is that in this case the poorly understood distribution of strange quarks within a proton gives no contribution. Hence, the amplitude of this production process is expressed exclusively in terms of the valence quark distributions. The relation between the electroproduction cross sections of $\pi, \eta, \eta^{\prime}$ in this reaction may be easily calculated in the same way as it was done above and one may prove that it exactly coincides with the one obtained in Eq.(39).

In conclusion, we have demonstrated that the ratios of cross sections of hard exclusive electroproduction of the neutral pseudoscalar mesons may be calculated in terms of the lowenergy physics. This low-energy physics crucially depends on the QCD axial anomaly and our results mean that one may explore the physics of spontaneous chiral symmetry breaking in the high-energy processes.

\section{ACKNOWLEDGMENTS}

We are deeply grateful to A. Radyushkin for useful discussion, to T. Feldmann for drawing our attention to the papers [24], and to S. Bass and M. Moinester for discovering a misprint in the first draft of the paper.

This work was supported by the U.S. Department of Energy Under Contract No. DEFG02-93ER-40771, and by the Israel Academy of Science under contract N 19-971. 


\section{REFERENCES}

[1] J. C. Collins, L. Frankfurt, and M. Strikman, Phys. Rev. D56 2982 (1997).

[2] L. Frankfurt and M. Strikman, hep-ph/9806536, Proceedings of DIS98 in press.

[3] J. F. Donoghue, E. Golowich, and B. R. Holstein, Dynamics of the Standard Model, Cambridge University Press, 1992.

[4] S. Weinberg, Phys. Rev., D11 3583 (1975).

[5] G. t'Hooft, Phys. Rev. Lett., 378 (1976).

[6] J. Schwinger, Phys. Rev., 82664 (1951); S. L. Adler, Phys. Rev., 1772426 (1968); J. S. Bell and R. Jackiw, Nuovo Cim., 60A 47 (1969).

[7] E. Witten, Nucl. Phys. B156, 269 (1979).

[8] G. Veneziano, Nucl. Phys. B159, 213 (1979).

[9] D. I. D'yakonov and M. I. Eides, Sov. Phys. JETP, 54232 (1981).

[10] S. J. Brodsky and G. P. Lepage, Phys. Lett. B87 359 (1979); Phys. Rev. Lett. 43545 (1979); 1625(E) (1979); Phys. Rev. D22, 2157 (1980).

[11] L. Frankfurt, A. Freund, V. Guzey, and M. Strikman, Phys.Lett. B418 345 (1998); B429 414(E) (1998).

[12] A.D. Martin and M.G. Ryskin, Phys. Rev. D57 6692 (1998).

[13] A. V. Radyushkin, Phys. Rev. D56 5524 (1997); preprint JLAB-THY-98-16, hepph/9805342, May 1998.

[14] V. Yu. Petrov, P. V. Pobylitsa, M. V. Polyakov, I. Bornig, K. Goeke, and C. Weiss, Phys.Rev. D57 4325 (1998).

[15] A. V. Efremov and A. V. Radyushkin, Phys. Lett. B94 245 (1980).

[16] V. L. Chernyak and A. R. Zhitnitsky, JETP Lett. 25510 (1977); Yad. Fiz. 311053 (1980).

[17] L.Frankfurt, W.Koepf, and M.Strikman, Phys.Rev.D54 3194 (1996), ibid D57 512 (1998).

[18] L. L. Frankfurt, G. A. Miller and M. Strikman, Ann. Rev. of Nucl. and Particle Phys. 44501 (1994).

[19] L. Mankiewicz, G. Piller, and T. Weig, preprint TUM/T39-97-28, hep-ph/9711227, 1997; Eur. Phys. J. C5 119 (1998).

[20] M. Vanderhaeghen, P.A.M. Guichon, M. Guidal, Phys. Rev. Lett. 805064 (1998).

[21] S. J. Brodsky, L. Frankfurt, J. F. Gunion, A. H. Mueller, and M. Strikman, Phys. Rev. D50 3134 (1994).

[22] A. Schafer, L. Mankiewicz, and O. Nachtmann, preprint UFTP-291-1992, published in DESY HERA Workshop 243 (1991); I. F. Ginzburg, D. Yu. Ivanov, and V. G. Serbo, Phys. At. Nucl. 561474 (1993).

[23] D. de Florian, O.A. Sampayo, and R. Sassot Phys.Rev. D57 5811 (1998).

[24] T. Feldmann and P. Kroll, Phys. Rev. D58 057501 (1998); T. Feldmann, P. Kroll, and B. Stech, preprint WU B 98-2, hep-ph/9802409; T. Feldmann and P. Kroll, Eur. Phys. J. C5 327 (1998).

[25] S. J. Brodsky and G. P. Lepage, Phys. Rev. D24, 1808 (1981).

[26] I. V. Musatov and A. V. Radyushkin, Phys. Rev. D56 2713 (1997). 


\section{FIGURES}

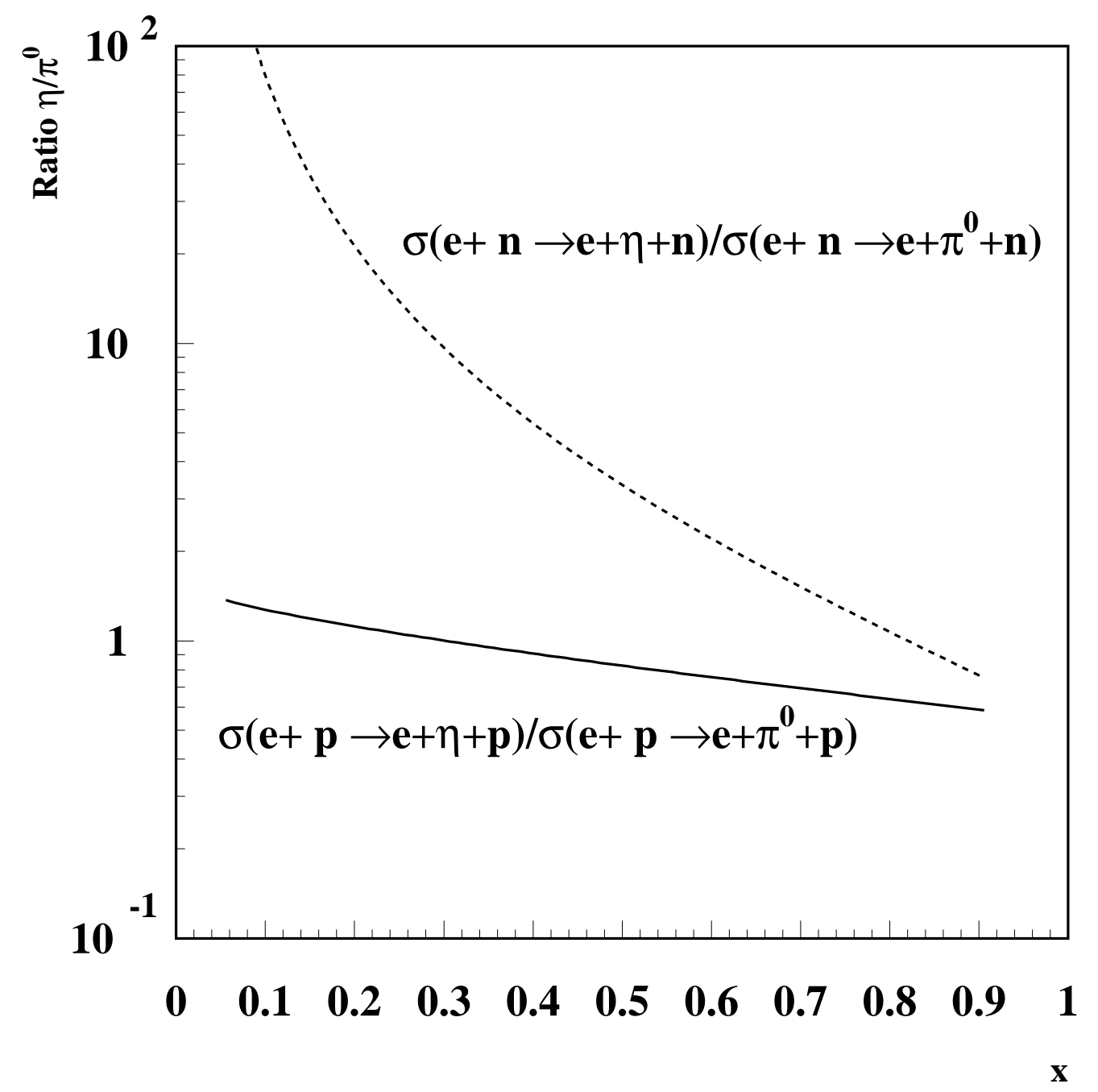

FIG. 1. The $x$ dependence of the ratio $\sigma\left(\gamma_{L}+N \rightarrow \eta+N\right) / \sigma\left(\gamma_{L}+N \rightarrow \pi^{0}+N\right)$ for the proton and neutron targets calculated using Eq.(38) and parameterization of $\Delta u_{V}$ and $\Delta d_{V}$ from [23]. 


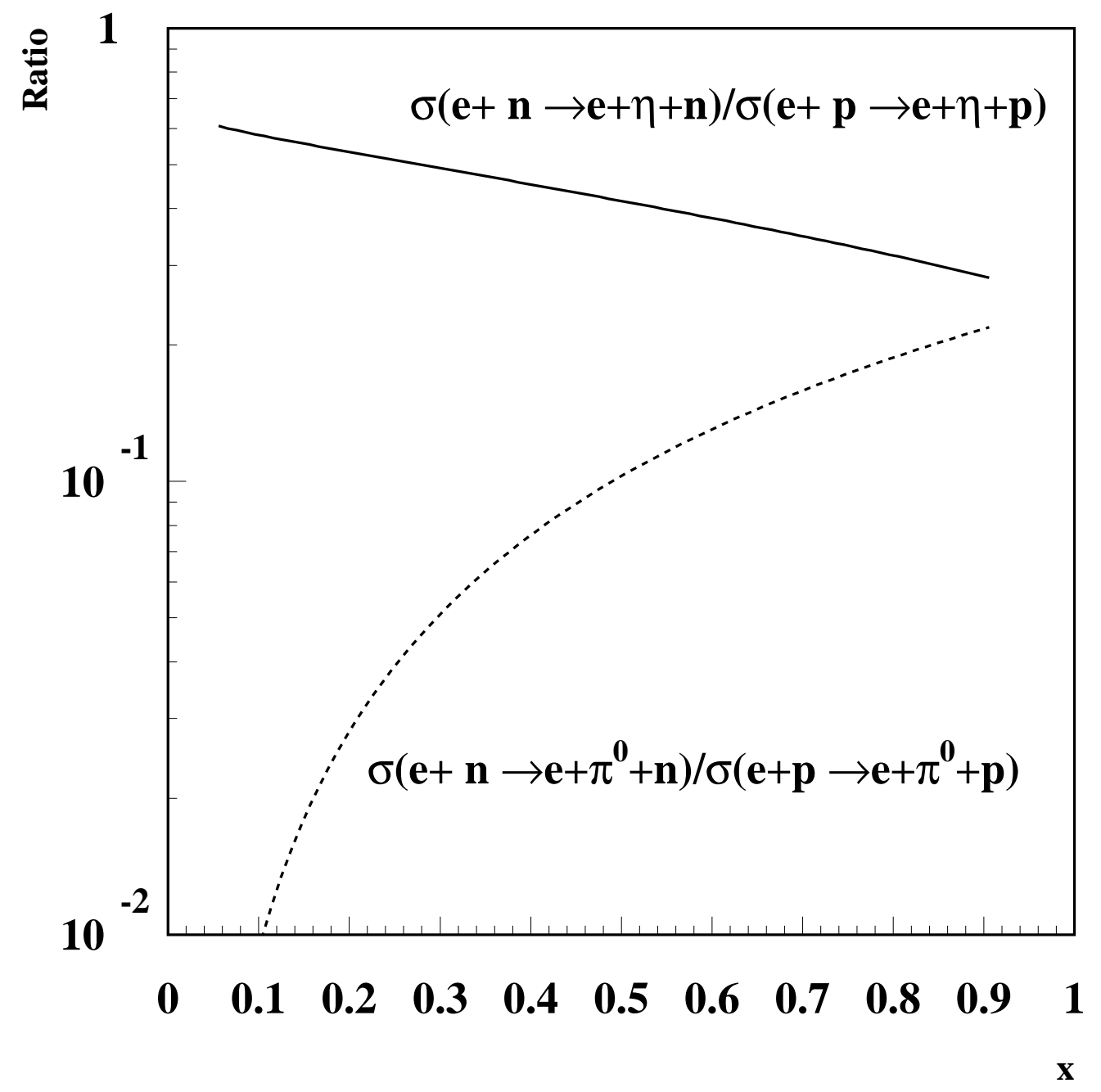

FIG. 2. The $x$ dependence of the ratio $\sigma\left(\gamma_{L}+n \rightarrow M+n\right) / \sigma\left(\gamma_{L}+p \rightarrow \pi^{0}+p\right)$ for production of $\eta$, and $\pi^{0}$ mesons calculated using Eq.(38) and parameterization of $\Delta u_{V}$ and $\Delta d_{V}$ from 23]. 